\title{
Restoring myelin, restoring nerve function
}

Loss of myelin underlies several disorders of the central nervous system (CNS) in humans, the most well known of which may be multiple sclerosis. Multiple sclerosis affects roughly 2.5 million people worldwide and can cause a range of symptoms including fatigue, loss of coordination and balance, numbness, blurred vision or blindness and even paralysis. It is thought to be an autoimmune disorder in which the immune system mistakenly attacks the body's own healthy tissue, destroying the myelin that surrounds nerve fibers of the CNS and interfering with transmission of nerve signals. There is no known cure for multiple sclerosis, although various treatments are available to help manage its symptoms and progression. Restoration of damaged myelin is thought to help protect nerve fibers from further degeneration and to restore signal transmission but, until now, had not been shown to result in functional recovery. New research from Ian Duncan (University of Wisconsin, Madison) and colleagues now suggests that remyelination can indeed

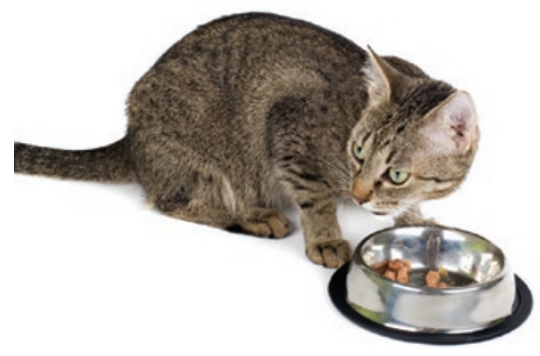

restore function in cats suffering from neurological demyelination.

Pregnant cats had been fed an irradiated diet as part of a study evaluating the effects of irradiated food on feline growth and development. After approximately 4 months on the irradiated diet, some of those cats developed severe progressive neurological dysfunction, including movement disorders, vision loss and paralysis. They were found to have widely distributed demyelination of the CNS, but nerve fibers remained intact. The cause of demyelination in the cats could not be identified. Biochemical analysis of the diet and of tissues from the affected cats showed no nutritional deficiencies or toxicities. The cats were put back on a normal diet and recovered slowly; after 2-4 months, they were neurologically normal. Examination of the CNS showed extensive remyelination, particularly in the optic nerve (Proc. Natl. Acad. Sci., published online 2 April 2009; doi:10.1073/ pnas.0812500106). The restored myelin sheath was thinner than normal but seemed to have normal physiological function.

The results of this study confirm that restoring damaged myelin in the CNS can restore function, provided that nerve fibers are preserved. "The fundamental point of the study is that it proves unequivocally that extensive remyelination can lead to recovery from a severe neurological disorder," explained Duncan. Duncan also noted that it is unlikely that irradiated food would have similar effects on human development, as the effects seem to be species-specific; pregnant rats fed an irradiated diet did not develop any neurological dysfunction.

Monica Harrington

\section{GOOD RTDDANCE TO BAD MEMORTES}

Though Hollywood has warned us that selectively erasing bad memories might have disastrous consequences, the prospect of forgetting traumatic experiences remains intriguing. A new study in mice suggests that this type of memory deletion might one day be possible, as researchers have managed to target the neurons involved in a single memory and to zap them-and the memory-into oblivion.

Scientists have long suspected that each memory can be traced to a particular group of neurons, but it has been difficult to isolate such neurons because they are scattered in different areas of the brain. In a previous study, however, Sheena Josselyn (Hospital for Sick Children and University of Toronto, Canada) and colleagues identified specific neurons that were involved in fear-related memories. When a mouse was trained to create a fear memory, neurons in the lateral amygdala with high expression of the protein CREB (cyclic adenosine monophosphate response element-binding protein) were more likely to be activated compared with other neurons.

In their new study, Josselyn and colleagues injected mice with a vector that caused some of their neurons to overexpress CREB and to become vulnerable to a specific toxin (Science 323, 1492-1496; 2009). Three days after injection, the researchers placed each mouse in a conditioning chamber and exposed it to a tone followed by a shock to the foot. This caused the mouse to create an auditory fear memory: ordinarily, a mouse exposed to the same tone a day later would immediately 'freeze' in anticipation of the shock. After the training session, the researchers injected the mouse with the toxin that killed neurons overexpressing CREB - neurons that would presumably have been recruited to create the new fear memory. When mice were exposed to the tone the following day, they showed no signs of fear, indicating that their fear memories had been deleted along with the neurons.

The fear memories seemed to have been erased completely and did not resurface over time. Killing the neurons did not seem to impair subjects' ability to acquire new fear memories; mice could be retrained to fear the shock. Furthermore, memories that were created before the injection of the vector were not affected.

The study sheds light on how memories are stored in the brain and shows that memory-specific neurons can be traced and manipulated. The ability to selectively eliminate fear memories might also have therapeutic applications, such as treatment of post-traumatic stress disorder. 